\title{
Sample-whitened matched filters
}

Andersen, Ib

\section{Published in:}

I E E E Transactions on Information Theory

Publication date:

1973

\section{Document Version}

Publisher's PDF, also known as Version of record

Link back to DTU Orbit

Citation (APA):

Andersen, I. (1973). Sample-whitened matched filters. I E E E Transactions on Information Theory, 19(5), 653660.

\section{General rights}

Copyright and moral rights for the publications made accessible in the public portal are retained by the authors and/or other copyright owners and it is a condition of accessing publications that users recognise and abide by the legal requirements associated with these rights.

- Users may download and print one copy of any publication from the public portal for the purpose of private study or research.

- You may not further distribute the material or use it for any profit-making activity or commercial gain

- You may freely distribute the URL identifying the publication in the public portal

If you believe that this document breaches copyright please contact us providing details, and we will remove access to the work immediately and investigate your claim. 


\title{
Sample-Whitened Matched Filters
}

\author{
IB N. ANDERSEN
}

\begin{abstract}
A sample-whitened matched filter (SWMF) for a channel with intersymbol interference and additive white Gaussian noise is defined as a linear filter with the properties that its output samples are a sufficient statistic for the MAP estimation of the transmitted sequence and have uncorrelated noise components. These filters are shown to exist for all realistic channels and the complete set of SWMF's for any channel is determined. It is shown that for nonpathological channels there is a unique SWMF which minimizes the amount of intersymbol interference defined as the discrete-time analog to the rms duration of a continuoustime signal. Finally, the theory is extended to more general modulation systems including pulse position modulation and frequency position modulation.
\end{abstract}

\section{INTRODUCTION}

$\mathrm{F}$ ORNEY [1] has recently obtained several interesting results for pulse-amplitude modulation (PAM) systems with intersymbol interference and additive white Gaussian noise (AWGN). In particular, when the intersymbol interference is of finite duration so that the channel autocorrelation function is a polynomial, Forney has shown that without loss of optimality the initial component of the receiver can be chosen as a linear filter, called a "whitened matched filter," with the property that the noise samples at the filter output are uncorrelated. Forney's synthesis procedure for this filter utilizes a spectral factorization of the pulse autocorrelation function and is easily extended to the case where this function is rational.

In this paper, we extend Forney's results in several directions. In Section II, we give a necessary and sufficient condition for filters with the properties of Forney's "whitened matched filter." In Section III, we prove that these filters exist in all cases of practical interest, and develop a synthesis procedure that requires only the extraction of the square root of a transfer function and that can be used to obtain every such filter. In Section IV, we extend the previous results to more general modulation systems. Finally, in Section V, we discuss how to choose between the possible filters, and in particular we develop a synthesis procedure for that filter with the properties of Forney's "whitened matched filter" which minimizes the amount of intersymbol interference at the filter output (defined as the discrete time analog to the rms duration of a continuoustime signal) and which may be the preferred receiving filter in a practical approximation to the optimum receiver.

\section{Sample-Whitened Matched Filters}

The transmitted signal in a PAM system as shown in Fig. 1 may be written

$$
s(t)=\sum_{k} x_{k} h(t-k T)
$$

Manuscript received July 21, 1972; revised December 18, 1972.

The author is with the Institute of Circuit Theory and Telecommunication, Technical University of Denmark, Lyngby, Denmark.

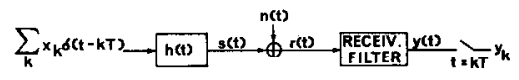

Fig. 1. PAM system.

where $h(t)$ is the overall channel impulse response, $x_{k}$ is the amplitude of the $k$ th-transmitted sample and is drawn from a finite alphabet, and $T$ is the symbol separation.

The received signal is given by $r(t)=s(t)+n(t)$, where $n(t)$ is AWGN. The PAM system has intersymbol interference if $h(k T)$ is nonzero for more than one integer $k$. The receiving filter is any linear filter, such that, its sampled outputs $y_{k}=y(k T)$ are a sufficient statistic for the maximum a posteriori probability (MAP) estimation of the data samples $x_{k}$ from $r(t)$. Following Forney, we define a samplewhitened matched filter (SWMF) as a receiving filter with the further property that the noise samples at its output are uncorrelated and hence statistically independent. (We prefer the terminology "sample-whitened matched filter" to Forney's "whitened matched filter" since the latter tends to suggest the incorrect conclusion that the noise process at the filter output is itself white noise. It is true, however, that if the output noise samples are further passed through an ideal low-pass filter, then the resultant process is low-pass white noise.)

Now let $w(-t)$ be the impulse response of any linear filter used in place of the receiving filter in Fig. 1. The noise samples at the output of this filter are given by

$$
n_{k}=\int n(t) w(t-k T) d t
$$

(integrations are taken from $-\infty$ to $\infty$ when the limits are unspecified). Letting $N_{0}$ be the one-sided noise power spectral density, we have for the correlation between samples

$$
\begin{aligned}
\overline{n_{j} n_{k}} & =\frac{N_{0}}{2} \iint \delta\left(t-t^{\prime}\right) w(t-k T) w\left(t^{\prime}-j T\right) d t^{\prime} d t \\
& =\frac{N_{0}}{2} \int w(t-k T) w(t-j T) d t .
\end{aligned}
$$

It follows from (2) that if we include an arbitrary normalization of $w(t)$, then a necessary and sufficient condition for the noise samples to be uncorrelated (or equivalently statistically independent since the noise process is Gaussian) is that

$$
\int w(t) w(t-k T) d t=\delta_{0 k}
$$

where $\delta_{0 k}$ is the Kronecker delta. Equation (3) is just the statement of orthonormality for the functions $w(t-k T)$.

Before proceeding to find a receiving filter satisfying (3) we have to discuss whatever is meant by MAP estimation of infinite sequences, since nothing was assumed about finiteness in the definition of a receiving filter. We say that 
the finite sequence $\hat{x}_{N}=\left(\hat{x}_{0}, \hat{x}_{1}, \cdots, \hat{x}_{N}\right)$ is a MAP estimate of a finite part of the transmitted sequence $x_{N}$ from a finite part of the actually received sequence $\boldsymbol{y}_{M}=\boldsymbol{\eta}_{M}=\left(\eta_{0}, \eta_{1}\right.$, $\left.\cdots, \eta_{M}\right)$ if

$$
P\left(\boldsymbol{x}_{N}=\hat{\boldsymbol{x}}_{N} \mid \boldsymbol{y}_{M}=\boldsymbol{\eta}_{M}\right) \geq P\left(\boldsymbol{x}_{N}=\xi_{N} \mid \boldsymbol{y}_{M}=\boldsymbol{\eta}_{M}\right)
$$

for any possible transmitted sequence $\xi_{N}$. For semi-infinite sequences these probabilities may not be defined, and MAP estimation must be defined in the sense of some limit. We define $\hat{\boldsymbol{x}}_{N}$ to be a MAP estimate of $\boldsymbol{x}_{N}$ from $\boldsymbol{\eta}=\left(\eta_{0}, \eta_{1}, \cdots\right)$ if there exists a $K$ such that (4) is satisfied for all $M>K$. In the cases of interest in this paper, the probabilities in (4) converge for $M \rightarrow \infty$, so that we can define

$$
P\left(x_{N}=\xi_{N} \mid \boldsymbol{y}=\boldsymbol{\eta}\right)=\lim _{M \rightarrow \infty} P\left(x_{N}=\xi_{N} \mid y_{M}=\eta_{M}\right)
$$

and write the MAP condition as

$$
P\left(x_{N}=\hat{x}_{N} \mid \boldsymbol{y}=\eta\right) \geq P\left(x_{N}=\xi_{N} \mid \boldsymbol{y}=\eta\right) .
$$

We now define $\tilde{x}_{p}$ to be the beginning of the MAP estimate of the entire sequence $\boldsymbol{x}$ from $\boldsymbol{y}=\boldsymbol{\eta}$ if there exists a $K$ such that all MAP estimates $\hat{\boldsymbol{x}}_{N}$ for $N>K$ begin with $\tilde{\boldsymbol{x}}_{p}$, i.e., $\hat{\boldsymbol{x}}_{N}=\left(\tilde{\boldsymbol{x}}_{p}, \hat{x}_{p+1}, \cdots, \hat{x}_{N}\right)$. This definition implies that a MAP estimate of the entire sequence may not exist since there need not exist a $K$ such that all $\hat{\boldsymbol{x}}_{N}$ have the same beginning for $N>K$. This concludes the definition of the MAP estimate $\hat{x}$ of $\boldsymbol{x}$ since an arbitrary symbol in $\hat{\boldsymbol{x}}$ is defined if possible by a sufficiently large beginning $\tilde{\boldsymbol{x}}_{p}$. The preceding definitions immediately extend to the case of infinite sequences $x=\left(\cdots, x_{-1}, x_{0}, x_{1}, \cdots\right)$ which permits us to deal with noncausal filters.

Now we can investigate when a filter $w(-t)$ satisfying (3) is also a true receiving filter. If the "impulse" input sequence $x_{k}=\delta_{0 k}$ is applied in Fig. 1, then the signal samples at the output of the filter $w(-t)$ are given by

$$
h_{k}=\int h(t) w(t-k T) d t
$$

which are just the projections of $h(t)$ on the orthonormal functions $w(t-k T)$. A necessary condition for the filter $w(-t)$ to be a receiving filter is that the signal $s(t)$, which in this case is $h(t)$, can be reconstructed from the received samples in the absence of noise, i.e., it must be true that

$$
h(t)=\sum_{k} h_{k} w(t-k T) .
$$

Equation (7) shows that the functions $w(t-k T), k$ integer, must be a basis for a space containing the functions $h(t-j T), j$ integer. This is also a sufficient condition for $w(-t)$ to be a true receiving filter since a component of $r(t)$ (see Fig. 1) orthogonal to this space is pure noise which by the whiteness assumption is statistically independent of any finite set of $y_{k}$ and hence irrelevant to the estimation of $\boldsymbol{x}_{N}$ from $\boldsymbol{y}_{M}[2, \mathrm{ch} .4]$. Consequently this component is irrelevant to the estimation of $x_{N}$ from $y$ and to the estimation of $x$ from $y$. We have proved the following theorem.

Theorem 1: The (normalized) filter $w(-t)$ is a samplewhitened matched filter for the channel $h(t)$ if and only if the functions $w(t-k T), k$ integer, are an orthonormal basis for a space containing the signal space spanned by the functions $h(t-k T), k$ integer, i.e., if and only if (3) and (7) are satisfied.

\section{EXISTENCE AND SYNTHESIS}

We now consider the questions of whether there exist filters $w(-t)$ such that (3) and (7) are satisfied, and, if so, how can these filters be found. In what follows, we assume that $h(t)$ and $w(t)$ have Fourier transforms, which we denote by $H(f)$ and $W(f)$, respectively. Applying Parseval's rule to (3), we obtain

$$
\int|W(f)|^{2} \exp (j 2 \pi f k T) d f=\delta_{0 k} .
$$

If we now interpret $|W(f)|^{2}$ as the transfer function of a channel, we see that (8) is just the statement that this channel must be free from intersymbol interference. A necessary and sufficient condition that $|W(f)|^{2}$ satisfy (8) is thus the Nyquist condition [3]-[5], namely

$$
\sum_{k}\left|W\left(f+\frac{k}{T}\right)\right|^{2}=T, \quad-\infty<f<\infty
$$

where the equality should be understood in the mean-square sense in $[-1 / 2 T, 1 / 2 T]$. A derivation of (9) is given in Appendix A. Transforming (7) we have further

$$
H(f)=W(f) \sum_{k} h_{k} \exp (-j 2 \pi f k T)
$$

from which we find

$$
\begin{aligned}
\sum_{k}\left|H\left(f+\frac{k}{T}\right)\right|^{2} & \\
& =\sum_{k}\left|W\left(f+\frac{k}{T}\right)\right|^{2}\left|\sum_{i} h_{i} \exp (-j 2 \pi i f T)\right|^{2} .
\end{aligned}
$$

With the aid of (9) this gives

$$
\sum_{k}\left|H\left(f+\frac{k}{T}\right)\right|^{2}=T\left|\sum_{i} h_{i} \exp (-j 2 \pi i f T)\right|^{2} .
$$

The complete solution of (11) is

$$
\sum_{i} h_{i} \exp (-j 2 \pi f i T)=\sqrt{\frac{P(f)}{T}} \exp [-j \beta(f)]
$$

where $\exp [-j \beta(f)], \beta(\cdot)$ real, is an arbitrary periodic function with period $1 / T$ and where we have defined $P(f)$ to be the periodic function

$$
P(f)=\sum_{k}\left|H\left(f+\frac{k}{T}\right)\right|^{2}
$$

which low-pass period is recognized to be the so-called "equivalent Nyquist transfer function" [4], for the original channel $h(t)$ cascaded with its time-reversal $h(-t)$ (the ordinary matched filter). $P(f)$ will be called the equivalent periodic power transfer function for $H(f)$. Moreover, we see that the solution (12) exists if and only if $\sqrt{P(f)} \exp$ $[-j \beta(f)]$ for some $\beta(\cdot)$ has a Fourier series expansion since the left-hand side of $(12)$ is the Fourier series expansion of the right. 
Finally, from (10) and (12) we have

$$
H(f)=W(f) \sqrt{\frac{P(f)}{T}} \exp [-j \beta(f)] .
$$

Equations (12) and (14) are necessary conditions for $W^{*}(f)$ to be a sample-whitened matched filter. In casc $P(f)$ has no zeros, these conditions are also sufficient as we see by solving (14) for $W(f)$,

$$
W(f)-H(f) \sqrt{\frac{T}{P(f)}} \operatorname{cxp}[j \beta(f)]
$$

and checking that this result satisfies (9) and (10) and hence (3) and (7). If $P(f)$ has zeros, (12) and (14) are no longer sufficient, since at a zero, say $f=f_{0},(14)$ does not uniquely specify $W\left(f_{0}\right)$ so that $W\left(f_{0}\right)$ must be chosen to satisfy (9). In this case, a set of sufficient conditions consists of (9), (12), and (14).

In all cases, satisfaction of (12) for some $\beta(\cdot)$ is sufficient for the existence of a SWMF as we will now show. Suppose that $P(f)$ is such that (12) can be satisfied for some $\beta(f)$. For $f$ 's such that $P(f) \neq 0, W(f)$ is determined by $(15)$. Next, we notice from the definition (13) of $P(f)$ that a zero of $P(f)$ must also be a zero of $H(f)$. This again implies, from (12), that (10) is satisfied for $f$ such that $P(f)=0$ regardless of the value of $W(f)$, and hence $W(f)$ can then be chosen to satisfy (9) at such $f$. If (13) is satisfied in the mean-square sense, then $W(f)$ defined in this way will satisfy (9) in the mean-square sense. The proof is given in Appendix B. We have now proved the following theorem.

Theorem 2: An SWMF for a channel $h(t)$ having an equivalent periodic power transfer function $P(f)$ in the mean-square sense exists if and only if $\sqrt{P(f)}$ can be expanded in a Fourier series. The complete set of SWMF's is the set of $W^{*}(f)$ 's satisfying (15) for $f$ such that $P(f) \neq 0$ and (9) when $P(f)=0$.

We note that $\beta(\cdot)$ should be chosen such that

$$
\exp [-j \beta(f)]=\exp [j \beta(-f)]
$$

if $w(t)$ is to be real. From (15), we see that if $P(f)$ has no zeros then $W^{*}(f)$ can be implemented as the cascade connection of the ordinary matched filter $H^{*}(f)$ and a transversal filter with transfer function $\sqrt{[T / P(f)]} \exp [-j \beta(f)]$.

$\Lambda t$ this point a connection can be made to Forney's [1] method of factorizing the so-called pulse autocorrelation function

$$
R(D)=\sum_{k} R_{k} D^{k}
$$

where the $R_{k}$ are defined by

$$
R_{k}=\int h(t) h(t-k T) d t
$$

Using Parseval's rule and rewriting the integral, we find

$$
\begin{aligned}
R_{k} & =\int|H(f)|^{2} \exp (+j 2 \pi f k T) d t \\
& =\int_{-1 / 2 T}^{1 / 2 T} P(f) \exp (+j 2 \pi f k T) d t
\end{aligned}
$$

i.e., $R_{k}$ is proportional to the $k$ th coefficient in the Fourier series expansion of $P(f)$. This gives us the relation

$$
P(f)=\frac{1}{T} R[\exp (-j 2 \pi f T)]
$$

from which it is evident that a factorization of $R(D)$ is equivalent to a factorization of $P(f)$. Furthermore, this relation justifies the adjective "spectral" used about the factorization of the "correlation" function $R(D)$, since $P(f)$ is a spectrum in the ordinary sense. If $R(D)$ has a factorization $R(D)=f(D) f\left(D^{-1}\right)$ then $f(\cdot)$ satisfies

$$
\begin{aligned}
f[\exp (-j 2 \pi f T)] & =\sqrt{T} \sum_{i} h_{i} \exp (-j 2 \pi f i T) \\
& =\sqrt{P(f)} \exp [-j \beta(f)]
\end{aligned}
$$

for somc $\beta(f)$, i.c.,

$$
f(D)=\sum_{i} \sqrt{T} h_{i} D^{i}
$$

where $\sqrt{T} h_{i}$ is the $i$ th coefficient in the Fourier series expansion of $\sqrt{P(f)} \exp [-j \beta(f)]$. The advantage of using Fourier transforms instead of $D$-transforms is that a SWMF has been proved to exist for any channel of practical interest and that such a filter is readily determined from Theorem 2.

\section{Extension to More General Modulation System}

We consider the case where communication takes place by means of amplitude modulation of $N$ standard pulses $h_{1}(t), \cdots, h_{N}(t)$ instead of only one pulse. Here the transmitted signal is

$$
s(t)=\sum_{i=1}^{N} \sum_{k} x_{i k} h_{i}(t-k T) .
$$

This model includes phase-modulated systems such as pulse- or frequency-position modulation, where the position (or frequency) of a pulse in each band is one of $N$ possible values. Generalizing the concept of a receiving filter to this more general case, we say that a bank of $M$ filters, $M \leq N$, are a set of receiving filters if their sampled outputs when the received signal $s(t)+n(t)$ is applied to each filter form sufficient statistics for the MAP estimation of the data samples $x_{i k}$. We want to find a set of rccciving filtcrs $w_{1}(-t), \cdots, w_{M}(-t), M \leq N$, that are also sample-whitening, i.e., such that the output noise samples are uncorrelated. Analogously with the derivation of Theorem 1, it is readily shown that a necessary and sufficient condition for such filters is that the functions $w_{1}(t-k T), \cdots, w_{M}(t-k T), k$ an integer, be an orthonormal basis for a space which includes the signal space spanned by the functions $h_{1}(t-k T), \cdots, h_{N}(t-k T), k$ an integer; i.e.,

$$
\int w_{i}(t) w_{j}(t-k T) d t=\delta_{0 k} \delta_{i j}
$$

and each of the standard pulses can be expanded as

$$
h_{i}(t)=\sum_{i=1}^{M} \sum_{k} h_{i l k} w_{l}(t-k T), \quad i=1, \cdots, N
$$




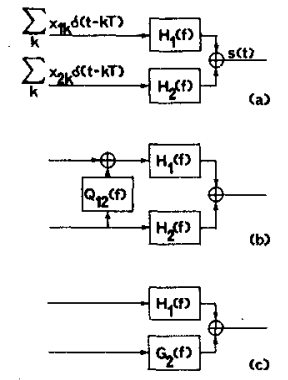

Fig. 2. Two waveform case. (a) Original system. (b) Periodic filter inserted. (c) Equivalent to (b) with $G_{2}(f)=H_{2}(f)+Q_{12}(f) H_{1}(f)$.

for some set of coefficients $h_{i l k}$. The receiving filters satisfying (16) and (17) can be found by a modified Gram-Schmidt procedure [2, ch. 4]. Consider first the case $N=2$, as shown in Fig. 2(a). $W_{1}(f)$ is found as $W(f)$ in Theorem 2 with $H(f)=H_{1}(f)$. Next, we notice that the insertion of a filter $Q_{12}(f)$ with periodic transfer function (a transversal filter), as indicated in Fig. 2(b), does not change the signal space. Hence, the system in Fig. 2(b) is equivalent to the one in Fig. 2(c) with the choice

$$
G_{2}(f)=H_{2}(f)+Q_{12}(f) H_{1}(f) .
$$

We then choose $Q_{12}(f)$ to make the signal spaces corresponding to $H_{1}(f)$ and $G_{2}(f)$ orthogonal, i.e., to satisfy

$$
\int H_{1}(f) G_{2}^{*}(f) \exp (j 2 \pi f k T) d f=0, \quad k \text { integer. }
$$

Using the same technique as in the derivation of the Nyquist condition (9) (writing an integral as a sum of integrals over intervals of length $T^{-1}$ and interchanging the order of summation and integration), we see that (18) is equivalent to

$\int_{-1 / 2 T}^{1 / 2 T}\left[Q_{12}(f) P_{1}(f)+P_{12}(f)\right] \exp (j 2 \pi f k T) d f=0$

where the periodic functions $P_{1}(f)$ and $P_{12}(f)$ are defined by

$$
\begin{aligned}
P_{1}(f) & =\sum_{k}\left|H_{1}\left(f+\frac{k}{T}\right)\right|^{2} \\
P_{12}(f) & =\sum_{k} H_{1}\left(f+\frac{k}{T}\right) H_{2}{ }^{*}\left(f+\frac{k}{T}\right) .
\end{aligned}
$$

The left side of (19) is the $k$ th coefficient in the Fourier series expansion of $Q_{12}(f) P_{1}(f)+P_{12}(f)$, and hence (18) is satisfied if and only if

$$
Q_{12}(f)=-\frac{P_{12}(f)}{P_{1}(f)} .
$$

(If $P_{1}(f)=0$, then $H_{1}(f+k / T)=0$ and $P_{12}(f)=0$. At such $f, Q_{12}(f)$ may be chosen arbitrarily.) Next, $W_{2}(f)$ is determined as $W(f)$ in Theorem 2 with $H(f)=G_{2}(f)$ (if $G_{2}(f)$ is not identically zero), and the resulting $W_{1}(f)$ and $W_{2}(f)$ satisfy $(16)$ and $(17)$.

This procedure extends in an obvious way for $N>2$. At the $i$ th step, we modify the channel $H_{i}(f)$ to a channel
$G_{i}(f)$ having a signal space orthogonal to the previous spaces. Then $W_{i}(f)$ is found as $W(f)$ in Theorem 2 with $H(f)=G_{i}(f)$.

\section{Cholce of Phase}

We have seen that the SWMF is never uniquely specified since the periodic phase factor exp $[j \beta(f)]$ in (12) may be chosen arbitrarily, and we now turn our attention to a particularly desirable $\beta(f)$. As Forney has noted [1], if the output samples of the SWMF are optimally processed (i.e., by a "Viterbi decoder" when the intersymbol interference has finite duration), then the energy per data sample can be used nearly as effectively as if there were no intersymbol interference. But as the complexity of the Viterbi decoder increases exponentially with this duration, it is desirable to concentrate the energy in the sampled impulse response in order to obtain a close approximation to the real response by a response of short duration, and at present there is no alternative for channels where the intersymbol interference has unlimited duration. In order to concentrate the energy we define the amount of intersymbol interference at the output of the SWMF, which we denote as $I$, as the timespread of the output samples in response to a single data sample; namely,

$$
I=\frac{\sum_{k}\left[\left(k T-t_{0}\right) h_{k}\right]^{2}}{T^{2} \sum_{i} h_{i}^{2}}
$$

where $t_{0}$ is the centroid of this response, i.e.,

$$
t_{0}=\frac{\sum_{k}(k T) h_{k}^{2}}{\sum_{i} h_{i}^{2}}
$$

and where the samples $h_{k}$ may be expressed in terms of $W(f)$ according to (6) as

$$
h_{k}=\int H(f) W^{*}(f) \exp (j 2 \pi f k T) d f .
$$

$I$ is the discrete-time analog to the rms duration [6] of a continuous-time signal $h(t)$ defined as $\int\left(t-t_{0}\right)^{2} h^{2}(t) d t$.

Our measure of intersymbol interference is somewhat arbitrary but it has the following "right" properties:

1) $I \geq 0$ with equality if and only if all the signal energy is concentrated in one sample; $(I=0$ is thus the ideal case of no intersymbol interference);

2) since the measure $I$ discriminates severely against the amplitude of pulse samples distant from the centroid $t_{0}$, minimizing $I$ will cluster the samples of significant amplitude near $t_{0}$ and thus simplify the optimum Forney receiver when the small samples are ignored;

3) $I$ is invariant to a change in $h(t)$ by, a constant gain factor and to a linear transformation of time;

4) this measure is mathematically tractable.

We now seek the SWMF that is "optimum" in the sense that it minimizes $I$. In an example we will show that this choice may not always be the best one, but in general it seems to be reasonably good. 
In the following $w(t)$ is assumed to be real. To minimize $I$, we differentiate (12) with respect to $f$ and obtain

$$
\begin{aligned}
-j 2 \pi \sum_{k} h_{k} k T \exp (-j 2 \pi f k T) & \\
= & \frac{1}{\sqrt{T}}\left[\frac{P^{\prime}(f)}{2 \sqrt{P(f)}} \exp [-j \beta(f)]\right. \\
& \left.-j \beta^{\prime}(f) \sqrt{P(f)} \exp [-j \beta(f)]\right]
\end{aligned}
$$

provided $P^{\prime}(f)$ exists and $P(f) \neq 0$ for all $f$. In what follows we will disregard such pathological exceptions. Squaring the absolute value and integrating from $-1 / 2 T$ to $1 / 2 T$ in (23), we obtain

$$
\sum_{k}\left(h_{k} k T\right)^{2}=\frac{1}{4 \pi^{2}} \int_{-1 / 2 T}^{1 / 2 T}\left[\frac{P^{\prime 2}(f)}{4 P(f)}+\beta^{\prime 2}(f) P(f)\right] d f .
$$

Similarly, integrating the product of (23) and the complex conjugate of (12) leads to

$$
\sum_{k} h_{k}^{2} k T=\frac{1}{2 \pi} \int_{-1 / 2 T}^{1 / 2 T} \beta^{\prime}(f) P(f) d f
$$

and integrating the square of the absolute value of (12) we have also

$$
\sum_{k} h_{k}^{2}=\int_{-1 / 2 T}^{1 / 2 T} P(f) d f
$$

Substituting (24)-(26) into (20) and using (21), we obtain

$$
\begin{aligned}
& I T^{2} \sum_{k} h_{k}{ }^{2} \\
& =\frac{1}{4 \pi^{2}} \int_{-1 / 2 T}^{1 / 2 T} \frac{P^{\prime 2}(f)}{4 P(f)} d f \\
& \quad+\frac{1}{4 \pi^{2}}\left[\int_{-1 / 2 T}^{1 / 2 T} \beta^{\prime 2}(f) P(f) d f-\frac{\left(\int_{-1 / 2 T}^{1 / 2 T} \beta^{\prime}(f) P(f) d f\right)^{2}}{\int_{-1 / 2 T}^{1 / 2 T} P(f) d f}\right]
\end{aligned}
$$

and we see that minimizing $I$ by choice of $\beta(f)$ is equivalent to minimizing the quantity in brackets in (27). Apart from a constant factor the term in brackets can be considered as the variance of a random variable $\beta^{\prime}(f)$ having a probability density function proportional to $P(f)$. From this consideration it also follows that the minimizing $\beta^{\prime}(f)$ is a constant. This constant can be expressed in terms of $t_{0}$ using (25) and (26). We find

$$
\frac{1}{2 \pi} \beta^{\prime}(f)=t_{0}, \quad \text { for all } f
$$

The left-hand side of this expression is the group delay of the filter $\exp [j \beta(f)]$ at frequency $f$, and we see that for the optimizing filter this delay must equal the average energy transmission time $t_{0}$ for all frequencies. The constraint that $\exp [-j \beta(f)]$ be periodic with period $1 / T$ means simply that $t_{0}$ must be one of the values

$$
t_{0}=k T, \quad k \text { integer. }
$$

We see that the optimizing filter is uniquely specified up to a delay that is an integer multiple of the symbol separation.
In the sequel $t_{0}$ will be taken as zero and the phase function $\beta(f)$ will be taken as identically zero. The corresponding $W(f)$ is denoted $W_{0}(f)$.

Besides being the SWMF that minimizes $I$, the filter

$$
W_{0}^{*}(f)=H^{*}(f) \sqrt{\frac{T}{P(f)}}
$$

is the SWMF that maximizes the fractional energy in the zeroth sample of the overall impulse response. This is evident from the expression

$$
h_{k}=T \int_{-1 / 2 T}^{1 / 2 T} \sqrt{\frac{P(f)}{T}} \exp [-j \beta(f)] \exp [+j 2 \pi f k T] d f
$$

with $k=0$. From this expression we also see that if $\exp [-j \beta(f)]=1$, then the response is symmetric, $h_{k}=$ $h_{-k}$, and the zeroth sample is the greatest one,

$$
\left|h_{0}\right|>\left|h_{k}\right|, \quad k=0 .
$$

These properties are common to the ordinary matched filter $H^{*}(f)$ and $W_{0}^{*}(f)$.

Finally, from (27), we see that the minimum attainable value of $I$ is

$$
I_{\min }=\frac{\frac{1}{16 \pi^{2}} \int_{-1 / 2 T}^{1 / 2 T} \frac{P^{\prime 2}(f)}{P(f)} d f}{T^{2} \int_{-1 / 2 T}^{1 / 2 T} P(f) d f} .
$$

Equation (29) may be interpreted as an extension of the Nyquist condition to channels with intersymbol interference, since it specifies the smallest amount of interference that can be attained on a channel with a given equivalent periodic power transfer function. The original Nyquist condition for freedom from intersymbol interference, i.e., $I_{\min }=0$, corresponds to $P^{\prime}(f)=0$, or equivalently $P(f)$ equals a constant, which is just (9).

SWMF's have been found to be optimum in another type of (suboptimum) receiver, namely a decision-feedback equalized receiver [7]. In this the optimum filter is the (unique) SWMF that yields a causal sampled response of the cascade connection of channel and receiving filter. This filter is obviously not the one with $\exp [j \beta(f)]=1$, and we shall now investigate how to choose the phase to make the sampled response causal. The derivation given below is similar to the one given for stochastic processes by Grenander and Rosenblatt [8].

The causality condition is

$$
h_{k}=0, \quad k<0 .
$$

We first assume that $\ln \sqrt{P(f)}$ has a Fourier series representation

$$
\ln \sqrt{P(f)}=\sum_{n} p_{n} \exp (-j 2 \pi f n T)
$$

and define a function

$$
g(D)=p_{0}+2 \sum_{n=1}^{\infty} p_{n} D^{n} .
$$


$g(D)$ is analytic inside the unit circle $|D|<1$ and the real part of it on the unit circle is

$$
\operatorname{Re} g[\exp (-j 2 \pi f T)]=\ln \sqrt{P(f)} .
$$

Furthermore, the function $f(D)=\exp [g(D)]$ is analytic in $|D|<1$ and its values on the unit circle are of the form

$$
f[\exp (-j 2 \pi f T)]=\sqrt{P(f)} \exp [-j \beta(f)]
$$

where we have defined the phase factor as

$$
\begin{aligned}
-j \beta(f) & =j \operatorname{Im} g[\exp (-j 2 \pi f T)] \\
& =\sum_{n=1}^{\infty} p_{n}[\exp (-j 2 \pi f n T)-\exp (j 2 \pi f n T)] .
\end{aligned}
$$

Since $f(D)$ is analytic in $|D|<1$, it has a series representation of the form

$$
f(D)=\sqrt{T} \sum_{k=0}^{\infty} h_{k} D^{k}
$$

that is convergent for $|D|<1$ and that if convergent for $|D|=1$ converges to the true value $\exp [g(D)]$. On the unit circle $D=\exp (-j 2 \pi f T)$, we have

$f(D)=\sqrt{P(f)} \exp [-j \beta(f)]=\sqrt{T} \sum_{k=0}^{\infty} h_{k} \exp (-j 2 \pi f k T)$

which after comparison to (12) shows that with the phase defined as above the sampled response is causal.

To cope with cases where $P(f)$ has zeros in such a way that $\ln \sqrt{P(f)}$ has no Fourier series, one can modify $P(f)$ to $P(f)+\varepsilon, \varepsilon>0$, in the preceding expressions. If the derivation works for any $\varepsilon>0$ then letting $\varepsilon \rightarrow 0$ will define a suitable phase. The difficulty in treating zeros of $P(f)$ reflects the fact that when an amplitude is zero the corresponding phase is undefined.

We notice that the SWMF giving a causal sampled response is identical to the filter resulting from Forney's "canonical spectral factorization" [1].

An Example: We shall consider a partial response class IV [9] system and compare Forney's SWMF [1] to ours with $\exp [j \beta(f)]=1$.

First we consider Forney's method. The channel impulse response is assumed to be

$$
h(t)=\left\{\begin{array}{cl}
\frac{1}{\sqrt{T}}, & 0<t<T \\
-\frac{1}{\sqrt{T}}, & 2 T<t<3 T \\
0, & \text { otherwise }
\end{array}\right.
$$

which is of finite duration so that Forney's method applies. This response is shown in Fig. 3(a). The chip $D$-transform of $h(t)$ is

$$
h(D, t)= \begin{cases}\frac{1}{\sqrt{T}}\left(1-D^{2}\right), & 0 \leq t<T . \\ 0, & \text { otherwise }\end{cases}
$$

and the pulse autocorrelation function is

$$
R(D)=-D^{-2}+2-D^{2}=\left(1-D^{2}\right)\left(1-D^{-2}\right)
$$
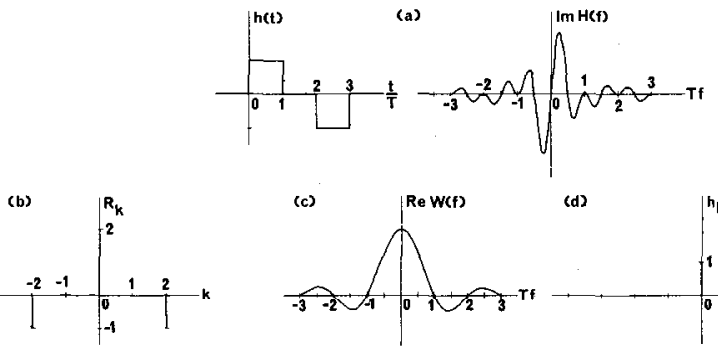

(d)
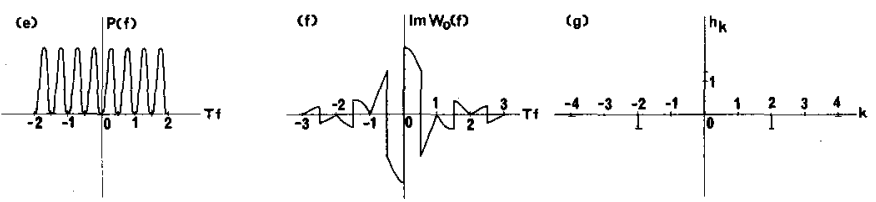

Fig. 3. Class IV partial response example. (a) Channel impulse response and transfer function. (b) Pulse autocorrelation function. (c) Ordinary $W(f)$. (d) Ordinary sampled response. (e) Equivalent periodic power transfer function. (f) $W(f)$ corresponding to $\exp [j \beta(f)]=1$. (g) Minimum $I$ sampled response.

which is factorized in the form

$$
R(D)=f(D) f\left(D^{-1}\right)
$$

The chip $D$-transform of Forney's $w(t)$ is

$$
w(D, t)=\frac{h(D, t)}{f(D)}= \begin{cases}\frac{1}{\sqrt{T}}, & 0 \leq t<T \\ 0, & \text { otherwise }\end{cases}
$$

which again implies

$$
w(t)= \begin{cases}\frac{1}{\sqrt{T}}, & 0 \leq t<T \\ 0, & \text { otherwise. }\end{cases}
$$

The sampled impulse response of $h(t)$ cascaded with $w(-t)$ is

$$
h(D)=\sum_{k} h_{k} D^{k}=1-D^{2}
$$

which is the ordinary class IV responsc.

The amount of interference in this response is $I=1$. This case is shown in Figs. 3(b)-(d).

Next we shall use the Fourier transform method. The channel transfer function is

$$
H(f)=2 \sqrt{T} j \sin (2 \pi f T) \operatorname{sinc}(\pi f T) \exp (-j 2 \pi f T)
$$

where

$$
\operatorname{sinc} x= \begin{cases}\frac{\sin x}{x}, & x \neq 0 \\ 1, & x=0 .\end{cases}
$$

The equivalent periodic power transfer function is found after some calculations, to be

$$
P(f)=4 T \sin ^{2}(2 \pi f T)
$$

and we have

$$
\begin{aligned}
W_{0}(f) & =H(f) \sqrt{\frac{T}{P(f)}} \\
& =j \sqrt{T} \frac{\sin 2 \pi f T}{|\sin 2 \pi f T|} \operatorname{sinc} \pi f T \exp (-j 2 \pi f T) .
\end{aligned}
$$


In this case the sampled impulse response is

$$
h(D)=\sum_{k \text { even }} \frac{4}{\pi} \frac{1}{1-k^{2}} D^{k}
$$

and the amount of interference is $I=1$. It is shown in Fig. 3(e)-(g). We notice that in both cases the amount of interference is the same, $I_{\min }=1$. This can occur because of the zeros in $P(f)$. Returning to the expression (27) for $I$, we see that introducing delta-impulses in $\beta^{\prime}(f)$ at the zeros of $P(f)$ will not affect the "variance" term since the zeros are of second order. This means that the minimizing phase is determined only up to discontinuities at the frequencies $k / 2 T, k$ integer. In agreement with this, the one SWMF can be transformed to the other by introduction of suitable phase discontinuities, see Fig. 3(c) and (f).

For use with a Viterbi decoder assuming a channel memory $M=2$ ( $M=$ number of time units from first to last nonzero pulse sample resulting from one single input impulse), clearly the ordinary response, Fig. 3(d), should be preferred since it has the memory $M=2$. For use with a simpler approximation to an optimum receiver, namely a simple linearly equalized receiver (which is a degenerate Viterbi decoder assuming $M=0$ ), the response in Fig. 3(g) will give the better performance since the useful fraction of the signal energy is

$$
\frac{h_{0}^{2}}{\sum_{k} h_{k}^{2}}=\frac{8}{\pi^{2}} \approx 81 \text { percent }
$$

while the remaining 19 percent is perceived as noise. The corresponding figures with the ordinary response are 50 percent and 50 percent, respectively.

However, we remark that in the linearly equalized case the receiving filter should not at all be a SWMF. Smith [10] has found the optimum receiving filter in the sense of maximizing the ratio between the energy in the zeroth pulse sample and the sum of the sampled-noise variance and the energy in the undesired pulse samples. Its transfer function can be expressed as $[4$, p. 112]

$$
\frac{\lambda H^{*}(f)}{1+\frac{d^{2}\left(L^{2}-1\right)}{3 N_{0}} P(f)}
$$

where $\lambda$ is a normalization constant, $L$ the number of amplitude levels used by the transmitter, and $2 d$ the spacing between them. Clearly this filter is not sample-whitened.

The example demonstrates that filters minimizing $I$ assure relatively good performance of receivers of arbitrary complexity whereas a better filter may be found for some specific receiver.

\section{Conclusion}

For any channel $H(f)$ the existence of a sample-whitened matched filter $W^{*}(f)$ for this channel was examined. It was found that for all channels of practical interest such filters exist, and the complete set of these filters was found. The theory was extended to more general modulation types than PAM. Finally, how to choose between the possible filters for different types of receivers was discussed. In particular it was examined how to choose the phase characteristic of the SWMF in order to minimize the amount of intersymbol interference, and an expression for the minimum attainable value of it was given.

\section{ACKNOWLEDGMENT}

The author wishes to express his gratitude to Prof. J. L. Massey who assisted in presenting these results in an easily understandable manner and who suggested the designations "sample-whitened matched filter" for $W^{*}(f)$ and "amount of intersymbol interference" for $I$.

\section{APPENDIX A}

\section{NYQuist CONDITION}

In [5], Gibby and Smith derive the Nyquist condition (9) for freedom from interference assuming uniform convergence of $\sum_{k} \mid W\left(f+k /\left.T\right|^{2}\right.$ in $[-1 / 2 T, 1 / 2 T]$. This assumption is too restrictive in some cases and here, we shall replace it by the assumption of convergence in the mean-square sense. We use the following.

Lemma: If $\left\{X_{N}(f)\right\}$ is a set of functions defined in a finite interval $I$ and $X(f)=1$.i.m. ${ }_{N \rightarrow \infty} X_{N}(f)$, then

$$
\int_{I} X(f) d f=\lim _{N \rightarrow \infty} \int_{I} X_{N}(f)
$$

The lemma simply states that if the sequence of $X_{N}(f)$ 's converges in the mean-square sense in a finite interval then it is permissible to interchange the order of the limit and the integration.

Proof: By definition $X(f)$ satisfies

$$
\int_{I}\left|X(f)-X_{N}(f)\right|^{2} d f<\varepsilon, \quad \text { for all } N>K
$$

where $K$ can be found for any $\varepsilon>0$. Application of Schwarz's inequality to the functions 1 and $\left|X(f)-X_{N}(f)\right|$ immediately shows

$$
\lim _{N \rightarrow \infty} \int\left|X(f)-X_{N}(f)\right| d f=0
$$

when the interval $I$ is finite, and finally we have

$$
0 \leq\left|\int_{I} X(f) d f-\int_{I} X_{N}(f) d f\right| \leq \int_{I}\left|X(f)-X_{N}(f)\right| d f
$$

which concludes the proof of the lemma.

We now use the lemma to rewrite the condition for freedom from interference

$$
\begin{aligned}
\delta_{0 k} & =\int|W(f)| \exp (j 2 \pi f k T) d f \\
& =\sum_{k} \int_{k / T-1 / 2 T}^{k / T+1 / 2 T}|W(f)|^{2} \exp (j 2 \pi f k T) d f \\
& =\sum_{k} \int_{-1 / 2 T}^{1 / 2 T}\left|W\left(f+\frac{k}{T}\right)\right|^{2} \exp (j 2 \pi f k T) d f \\
& =\int_{-1 / 2 T}^{1 / 2 T}\left(\sum_{k}\left|W\left(f+\frac{k}{T}\right)\right|^{2}\right) \exp (j 2 \pi f k T) d f .
\end{aligned}
$$

Since the functions $\exp (j 2 \pi f k T)$ are a complete orthogonal basis for functions in $[-1 / 2 T, 1 / 2 T]$ the preceding equation can 
be satisfied if and only if

$$
T=\lim _{N, M \rightarrow \infty} \sum_{k=-M}^{N}\left|W\left(f+\frac{k}{T}\right)\right|^{2}, \quad|f| \leq 1 / 2 T
$$

which is the strict formulation of (9).

\section{APPENDIX B}

In here, we prove that convergence in the mean-square sense of $\sum_{k}|H(f+k / T)|^{2}$ implies convergence of $\sum_{k}|W(f+k / T)|^{2}$ as stated in Theorem 2. We have to show that

$$
P(f)=\lim _{N, M \rightarrow \infty} \sum_{-M}^{N}\left|H\left(f+\frac{k}{T}\right)\right|^{2}
$$

implies that

$$
\left.\left.\lim _{N, M \rightarrow \infty} \int_{-1 / 2 T}^{1 / 2 T}\left|T-\sum_{-M}^{N}\right| W\left(f+\frac{k}{T}\right)\right|^{2}\right|^{2} d f=0
$$

where $W(f)$ satisfies (15) when $P(f)=0$ and (9) otherwise. To this purpose we write the error integral as the sum of three terms

$$
\begin{aligned}
\left.\left.\int_{-1 / 2 T}^{1 / 2 T}\left|T-\sum_{-M}^{N}\right| W\left(f+\frac{k}{T}\right)\right|^{2}\right|^{2} d f & \\
& =\int_{f: P=0}+\int_{f: 0<P<\delta}+\int_{f: \delta \leq P}
\end{aligned}
$$

where $\delta$ is an arbitrary nonnegative constant. The first of these integrals is immediately seen to converge to zero since we have simply defined $W(f)$ to satisfy $(9)$ when $P(f)=0$.

In the second and third integral $W(f)$ is defined by (15), and the integrand apart from the factor $1 / T^{2}$ is

$$
\left|\frac{1}{P(f)}\left(P(f)-\sum_{-M}^{N}\left|H\left(f+\frac{k}{T}\right)\right|^{2}\right)\right|^{2} \leq 1 .
$$

The second integral is consequently overbounded by

$$
\frac{1}{T^{2}} \int_{f: 0<P<\delta} 1 d f
$$

and since no fixed value $P(f)>0$ can satisfy $0<P(f)<\delta$ for arbitrarily small $\delta$ this integral can be made arbitrarily small by choice of $\delta$.

Since the integrand is less than 1 the third integral is overbounded by

$$
\int_{f: \delta \leq P}\left|\frac{1}{P(f)}\left(P(f)-\sum_{-M}^{N}\left|H\left(f+\frac{k}{T}\right)\right|^{2}\right)\right| d f .
$$

Now we apply Schwarz's inequality to obtain the upper bound

$$
\begin{aligned}
\sqrt{\left.\left.\int_{f: \delta \leq P} \frac{1}{P^{2}(f)} d f \int_{f: \delta \leq P}\left|P(f)-\sum_{-M}^{N}\right| H\left(f+\frac{k}{T}\right)\right|^{2}\right|^{2} d f} \\
\leq \sqrt{\left.\left.\frac{1}{T \delta^{2}} \int_{-1 / 2 T}^{1 / 2 T}\left|P(f)-\sum_{-M}^{N}\right| H\left(f+\frac{k}{T}\right)\right|^{2}\right|^{2} d f}
\end{aligned}
$$

which can be made arbitrarily small for any $\delta$ by choice of $N$ and $M$ when $\sum_{k}|H(f+k / T)|^{2}$ converges in the mean-square sense. We have now proved that the existence of $P(f)$ in the mean-square sense implies the existence of $W(f)$.

\section{REFERENCES}

[1] G. D. Forney, Jr., "Maximum likelihood sequence estimation of digital sequences in the presence of intersymbol interference," IEEE Trans. Inform. Theory, vol. IT-18, pp. 363-378, May 1972.

[2] J. M. Wozencraft and I. M. Jacobs, Principles of Communication Engineering. New York: Wiley, 1965.

[3] H. Nyquist, "Certain topics in telegraph transmission theory," Trans. AIEE, vol. 47, pp. 617-644, Apr. 1928.

[4] R. W. Lucky, J. Salz, and E. J. Weldon, Jr., Principles of Data Communication. New York: McGraw-Hill, 1968, ch. 4.

[5] R. A. Gibby and J. W. Smith, "Some extensions of Nyquist's tclcgraph transmission theory," Bell Syst. Tech. J., vol. XLIV, pp. 1487-1510, Sept. 1965.

[6] A. Papoulis, The Fourier Integral and its Applications. New York: McGraw-Hill, 1962, p. 62.

[7] R. Price, "Nonlinearly feedback-equalized PAM versus capacity for noisy filter channels," preprint 1972 Int. Conf. Communication.

[8] U. Grenander and M. Rosenblatt, Statistical Analysis of Stationary Time Series. New York: Wiley, 1957, ch. 2.

[9] E. R. Kretzmer, "Generalization of a technique for binary data communication," IEEE Trans. Commun. Technol. (Commun.), vol. COM-13, pp. 67-68, Feb. 1966.

[10] J. W. Smith, "The joint optimization of transmitted signal and receiving filter for data transmission systems," Bell Syst. Tech.J., vol. XLIV, pp. 2363-2392, Dec. 1965. 